\title{
Holiday Effect of East Asian Markets Reciprocally
}

\author{
Beenish Bashir and Shumaila Zeb
}

\begin{abstract}
This paper reconnoitered the holiday effect of East Asian stock markets over each other. SSE-180 index (Shanghai Stock Exchange), NIKKIE 225 (Japan), TWII (Taiwan Weighted Index), HSI (Hang Seng) epitomized East Asian region. The key objective is to investigate the return effect on East Asian stock markets coinciding with the S\&P 500 (Standard and poor) holidays further the return effect on East Asian stock markets during the trading session when there is no trading on other East Asian stock markets. By means of TGARCH model using daily return of East Asian Stock Exchanges and S\&P 500 from January 1, 2003 to December 31, 2012.Day-of-the- Week effect, as well as regional and international spillovers has been considered, robust results have been found by this study. Outcomes of this paper have implications for international investors. It is of immense importance for an investor to consider the holidays of the interlinked stock markets when investing in a particular market of a region as holidays not only affect the returns of the portfolios but risk as well.
\end{abstract}

Index Terms-Holiday effect, international spillovers, TGARCH model, volatility.

\section{INTRODUCTION}

Globalization has made the worldwide financial markets more interconnected and linked, an indulgence of the correlations and interactions among various financial markets is essential for financial institutions, investors, and governments. Capital market liberalization results into a high correlation amongst local and international markets [1]. East Asian countries are considered for studying the interconnection. As, economy of this region is rising being the home of world's most affluent economies like China, Taiwan, Japan and Hong Kong. "Reference [2] shows that East Asia contributed $40 \%$ of world growth and the regional growth was $7.5 \%$. It is anticipated that in 2013 this regional growth will raise to $7.8 \%$ ". This research focus on stock markets and efficient market hypothesis (EMH) is the most vital concept for such analysis. Financial markets are information-ally efficient and market prices completely reflect available information [3]. However, seasonal effects are one of the prominent anomalous return trends, specifically, day-of-the-week effect, pre-holiday effect [4]. Further reference [5] explored significant pre-holiday effects in the New Zealand market; and reference [6] documented pre-holiday effect in Australia. Studies are also conducted to investigate the information integration in these markets, various stock market indices of Asia, Europe and US market to explore how these markets are linked and how information flow from one market to another market holiday. The lag of

Manuscript received September 12, 2013; revised November 18, 2013.

Beenish Bashir is with the NUST Business School, National University of Sciences and Technology, Islamabad, Pakistan (e-mail: beenish128@hotmail.com).
Asian market is found statistical significant for the flow of holiday information because of the time zone difference in developed countries sock markets and emerging markets [7]. Similar results are found in future market [8]. There is also an impact of US holiday on other global markets. On US holiday, European market expects to get average return, because of unavailability of US investor and lack of macroeconomic information. These out comes of US holiday will change the investor mix on European markets [9]. The data comprise of the daily opening and closing of five indexes: SSE-180 index (China), NIKKIE 225 (Japan), TWII (Taiwan), HSI (Hong Kong) and S\&P 500 (US). These indexes reflect overall stock market activities of selected countries. Using opening and closing prices, we have obtained two return measures: The ordinary return $\left(R_{t}^{c c}\right.$ close to close returns) and the intraday return $\left(R_{t}^{o c}\right.$ open to close return).

\section{MEthodology}

In this study we aim to model the returns of selected indexes, with conditional volatility models. Results given in this paper are from TGARCH model by using the following TGARCH model:

$$
\begin{aligned}
r_{t}^{i}=\alpha_{0}+\emptyset_{1} r_{t-1}^{i} & +\sum_{i=1}^{4} \alpha_{i} D_{o} w_{i t}+\sum_{i=1}^{3} \beta_{i} r_{t}^{i}+\delta_{1} r_{t-1}^{u s} \\
& +\sum_{i=1}^{4} \rho_{i} p D_{h}+\sum_{i=1}^{4} \xi_{i} n D_{h}+\varepsilon_{t}
\end{aligned}
$$

where

$\varepsilon_{t}$ follows a $N\left(0, h_{t}\right)$;

$$
\begin{gathered}
h_{t}=\omega+\gamma_{1} \varepsilon_{t-1}^{2}+\gamma_{2} h_{t-1}+\gamma_{3} I_{t} \varepsilon_{t-1}^{2}+ \\
\sum_{i=1}^{4} v_{i} D_{t} I_{t} \varepsilon_{t-1}^{2}+\sum_{i=1}^{4} \theta_{i} \varepsilon_{t-1}^{2} \\
I_{t}=\left\{\begin{array}{l}
1 \text { if } \varepsilon_{t-1}<0 \\
0 \text { if } \varepsilon_{t-1} \geq 0
\end{array}\right. \\
D_{t}=\left\{\begin{array}{c}
1 \text { if there is market off in country } i \\
0 \text { otherwise }
\end{array}\right.
\end{gathered}
$$

Refer to "(1)", $\sum_{i=1}^{4} \alpha_{i} D_{o} w_{i t}$ is dummy variable for the Day-of-the Week with value " 1 " for Tuesday, Wednesday, Thursday and Friday, $i=1 \ldots 4 . \emptyset_{1} r_{t-1}^{i}$ is the lagged term of index on the left side of the equation which identify serial correlation in the data and also tell us whether return of day 1 affects the return of day $2 i$-e next day returns. $\sum_{i=1}^{3} \beta_{i} r_{t}^{i}$ is the return of remaining three East Asian markets when the fourth one is acting as $r_{t}^{i}, i=1,2,3$. As US is 12 hours behind the East Asian time zone, lagged term of US return $r_{t-1}^{u s}$ is considered [6]. $\sum_{i=1}^{4} \rho_{i} D_{h}$ is the dummy variable for 
positive US holiday after positive closure of S\&P 500 and for three regional countries on the right side of mean equation when one of the regional country is on the left side of the mean equation, $i=1 \ldots 4$. This dummy variable takes the value of " 1 " on positive US holiday and on the positive holiday of three regional countries otherwise " 0 ". $\sum_{i=1}^{4} \xi_{i} n D_{h}$ is the dummy variable for negative US holiday after negative closure of S\&P 500 and for three regional countries on the right side of mean equation when one of the regional country is on the left side of the mean equation, $i=1 \ldots 4$. This dummy variable takes the value of " 1 " on negative US holiday and on the negative holiday of three regional countries otherwise " 0 ". Conditional volatility equation is the form of asymmetric GARCH model [10]. Refer to "(2)", checks impact of positive and negative innovation on volatility by using dummy variable $I_{t}$. This dummy variable is " 1 " if $\varepsilon_{t-1}<0$ otherwise " 0 ". The positive and significant value of this dummy depicts that non-positive jolt has more impact as compare to the positive jolt of the equal extent. $\sum_{i=1}^{p} \theta i \varepsilon_{t-1}^{2}$ are lag of squared error terms of Taiwan, Hong Kong, Japan and US indices to testify volatility transmission from regional and international markets. If_- $v_{i}<0$ then it is concluded that holiday has a reducing effect on the volatility. If $=0$ then there is no impact of holiday on market volatility and if $>0$ then holiday increased the market volatility. Holiday is modeled through dummy variable holiday. This dummy is " 1 " on holiday of US and East Asian markets and " 0 " otherwise.

TABLE I: DESCRIPTIVE STATISTICS

\begin{tabular}{|c|c|c|c|c|c|c|}
\hline \multicolumn{2}{|c|}{ Statistics } & \multirow{2}{*}{$\begin{array}{c}\text { Hong Kong } \\
0.000339\end{array}$} & \multirow{2}{*}{$\begin{array}{c}\text { Japan } \\
6.76 \mathrm{E}-05\end{array}$} & \multirow{2}{*}{$\begin{array}{c}\text { China } \\
0.000254\end{array}$} & \multirow{2}{*}{$\begin{array}{c}\text { Taiwan } \\
0.000204\end{array}$} & \multirow{2}{*}{$\begin{array}{c}\text { USA } \\
0.000204\end{array}$} \\
\hline Mean & $\mathrm{CC}$ & & & & & \\
\hline & OC & -0.000188 & -0.000315 & 0.000818 & -0.001245 & 0.000243 \\
\hline \multirow[t]{2}{*}{ Median } & $\mathrm{CC}$ & 0.000000 & 0.000000 & 0.000000 & 5.37E-05 & 0.000452 \\
\hline & $\mathrm{OC}$ & 0.000000 & 0.000000 & 0.000000 & -0.000506 & 0.000506 \\
\hline \multirow[t]{2}{*}{ Maximum } & $\mathrm{CC}$ & 0.134068 & 0.132346 & 0.107116 & 0.065246 & 0.109572 \\
\hline & $\mathrm{OC}$ & 0.121553 & 0.116581 & 0.084430 & 0.059570 & 0.102457 \\
\hline \multirow[t]{2}{*}{ Minimum } & $\mathrm{CC}$ & -0.135820 & -0.121110 & -0.105661 & -0.069123 & -0.094695 \\
\hline & $\mathrm{OC}$ & -0.116162 & -0.105634 & -0.101384 & -0.055916 & -0.091272 \\
\hline \multirow[t]{2}{*}{ SD } & $\mathrm{CC}$ & 0.015687 & 0.014939 & 0.017304 & 0.013252 & 0.012882 \\
\hline & $\mathrm{OC}$ & 0.011019 & 0.011359 & 0.016017 & 0.010326 & 0.012446 \\
\hline \multirow[t]{2}{*}{ Skewness } & $\mathrm{CC}$ & 0.034131 & -0.582261 & -0.303955 & -0.366019 & -0.299995 \\
\hline & OC & 0.293570 & -0.596340 & -0.244074 & -0.133175 & -0.352445 \\
\hline \multirow[t]{2}{*}{ Kurtosis } & $\mathrm{CC}$ & 12.78474 & 12.56809 & 7.504199 & 6.133648 & 13.64460 \\
\hline & $\mathrm{OC}$ & 18.69388 & 18.69388 & 6.276953 & 6.168689 & 13.17956 \\
\hline \multirow[t]{2}{*}{ Jarque-Bera } & $\mathrm{CC}$ & 10408.36 & 10099.48 & 2245.628 & 1125.743 & 12356.61 \\
\hline & $\mathrm{OC}$ & 26133.85 & 28945.85 & 1163.074 & 1071.401 & 11032.42 \\
\hline \multirow[t]{2}{*}{ Probability } & $\mathrm{CC}$ & $0.000000^{* * *}$ & $0.000000 * * *$ & $0.000000 * * *$ & $0.000000 * * *$ & $0.000000 * * *$ \\
\hline & $\mathrm{OC}$ & $0.000000 * * *$ & $0.000000 * * *$ & $0.000000 * * *$ & $0.000000 * * *$ & $0.000000 * * *$ \\
\hline \multirow[t]{2}{*}{ Sum } & $\mathrm{CC}$ & 0.883430 & 0.176488 & 0.661943 & 0.531567 & 0.450384 \\
\hline & $\mathrm{OC}$ & -0.477777 & -0.801849 & 2.080440 & -3.167244 & 0.617163 \\
\hline
\end{tabular}

TABLE II: CORRELATION MATRIX-ORDINARY RETURN

\begin{tabular}{|c|c|c|c|c|c|}
\hline Correlation(CC) & Hong Kong & Japan & China & Taiwan & USA \\
\hline Hong Kong & 1.000000 & & & & \\
\hline Japan & $\begin{array}{c}0.608600^{* * *} \\
(0.0000)\end{array}$ & 1.000000 & & & \\
\hline China & $\begin{array}{c}0.404672^{* * *} \\
(0.0000)\end{array}$ & $\begin{array}{c}0.239845^{* * *} \\
(0.0000)\end{array}$ & 1.000000 & & \\
\hline Taiwan & $\begin{array}{c}0.569828^{* * *} \\
(0.0000)\end{array}$ & $\begin{array}{c}0.548801^{* * *} \\
(0.0000)\end{array}$ & $\begin{array}{c}0.246439^{* * *} \\
(0.0000)\end{array}$ & 1.000000 & \\
\hline USA & $\begin{array}{c}0.224620^{* * *} \\
(0.0000)\end{array}$ & $\begin{array}{c}0.115615^{* * *} \\
(0.0000)\end{array}$ & $\begin{array}{c}0.066550 * * * \\
(0.0000)\end{array}$ & $\begin{array}{c}0.129891^{* * *} \\
(0.0000)\end{array}$ & 1.000000 \\
\hline
\end{tabular}

TABLE III: CORRELATION MATRIX-INTRADAY RETURN

\begin{tabular}{|c|c|c|c|c|c|c|}
\hline Correlation(OC) & Hong Kong & Japan & China & Taiwan & USA & \\
\hline Hong Kong & 1.000000 & & & & & \\
\hline Japan & $\begin{array}{l}0.306383 * * * \\
(0.0000)\end{array}$ & 1.000000 & & & & \\
\hline China & $\begin{array}{l}0.360209 * * * \\
(0.0000)\end{array}$ & $\begin{array}{l}0.135094 * * * \\
(0.0000)\end{array}$ & & 0000 & & \\
\hline Taiwan & $\begin{array}{l}0.422690 * * * \\
(0.0000)\end{array}$ & $\begin{array}{l}0.287504 * * * \\
(0.0000)\end{array}$ & $\begin{array}{l}0.199 \\
(0.00\end{array}$ & $17 * * *$ & 1.000000 & \\
\hline USA & $\begin{array}{l}0.310153 * * * \\
(0.0000)\end{array}$ & $\begin{array}{l}0.136243 * * * \\
(0.0000)\end{array}$ & $\begin{array}{l}0.081 \\
(0.00\end{array}$ & $11 * * *$ & $\begin{array}{l}0.179563 * * * \\
(0.0000)\end{array}$ & 1.000000 \\
\hline
\end{tabular}


TABLE IV (A): ESTIMATION- ORDINARY RETURN

\begin{tabular}{|c|c|c|c|c|c|c|c|c|c|c|c|c|c|c|c|c|c|c|c|c|c|}
\hline$\tilde{\Sigma}^{h}$ & 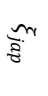 & $\begin{array}{l}\text { ज्ञ } \\
\text { है }\end{array}$ & $\widetilde{\Xi}^{n}$ & $\begin{array}{l}\widetilde{W} \\
\tilde{b}_{0}^{n}\end{array}$ & $D_{\tilde{\xi}}^{D}$ & $\begin{array}{l}\text { Dे } \\
\text { छी }\end{array}$ & 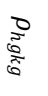 & D & $\begin{array}{l}\overline{0} \\
\vdots \\
\infty \\
\delta\end{array}$ & $\infty$ & ఫొ & 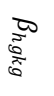 & $\Phi$ & $\begin{array}{l}\text { పు } \\
6 \\
6\end{array}$ & $\stackrel{8}{2}$ & $\omega$ & న & $\beta$ & $\theta$ & 8 & 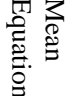 \\
\hline $\begin{array}{l}\dot{0} \\
\dot{8} \\
0 \\
0 \\
0 \\
\dot{0}\end{array}$ & $\begin{array}{l}\stackrel{0}{0} \\
\dot{0} \\
0 \\
\infty \\
\infty \\
\infty\end{array}$ & $\begin{array}{l}1 \\
0 \\
8 \\
8 \\
0 \\
0 \\
\dot{N} \\
\stackrel{N}{ }\end{array}$ & 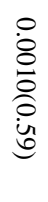 & & 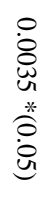 & 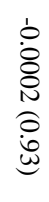 & 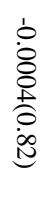 & $\begin{array}{l}\stackrel{0}{0} \\
\stackrel{0}{0} \\
\text { Oे } \\
\dot{0}\end{array}$ & & 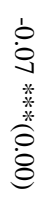 & 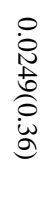 & 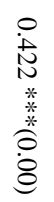 & 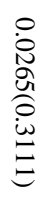 & & 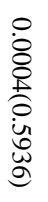 & \begin{tabular}{l}
$\dot{b}$ \\
$\dot{8}$ \\
0 \\
0 \\
$*$ \\
\multirow{2}{*}{} \\
$\dot{0}$ \\
$\dot{E}$
\end{tabular} & $\begin{array}{l}\stackrel{0}{0} \\
\dot{0} \\
\stackrel{\omega}{0} \\
\stackrel{0}{0} \\
\dot{d}\end{array}$ & $\begin{array}{l}\dot{b} \\
\dot{8} \\
\stackrel{0}{0} \\
\dot{0}\end{array}$ & $\begin{array}{l}\stackrel{0}{0} \\
\dot{8} \\
\text { O্ } \\
\text { Oे } \\
\dot{\vec{d}}\end{array}$ & $\begin{array}{l}\dot{0} \\
\dot{8} \\
\text { W. } \\
\text { : } \\
\dot{0}\end{array}$ & $\overrightarrow{F_{0}}$ \\
\hline $\begin{array}{l}\circ \\
\text { o } \\
\text { Oे } \\
\text { fo }\end{array}$ & $\begin{array}{l}0 \\
\dot{8} \\
0 \\
0 \\
0 \\
\text { i্ }\end{array}$ & & $\begin{array}{l}\dot{1} \\
\dot{8} \\
\stackrel{0}{0} \\
\text { i্ }\end{array}$ & $\begin{array}{l}\dot{0} \\
\dot{8} \\
\stackrel{0}{0} \\
\dot{0}\end{array}$ & $\begin{array}{l}\stackrel{0}{8} \\
\stackrel{0}{\circ} \\
\dot{\Xi}\end{array}$ & $\begin{array}{l}\dot{0} \\
\dot{8} \\
\stackrel{0}{0} \\
\dot{U}\end{array}$ & & 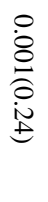 & 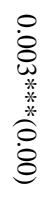 & 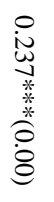 & 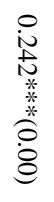 & & 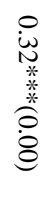 & 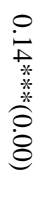 & $\begin{array}{l}\dot{b} \\
\dot{0} \\
0 \\
0 \\
\text { :̆ } \\
0\end{array}$ & $\begin{array}{l}\dot{b} \\
\dot{8} \\
0 \\
0 \\
\dot{d} \\
0\end{array}$ & 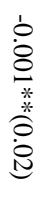 & $\begin{array}{l}\dot{0} \\
\dot{8} \\
\infty \\
\stackrel{*}{0} \\
\dot{0} \\
\dot{0}\end{array}$ & 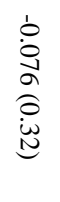 & 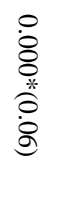 & 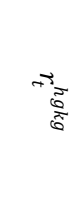 \\
\hline 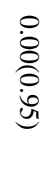 & & $\begin{array}{l}\dot{0} \\
\dot{8} \\
\stackrel{0}{0} \\
\dot{\infty}\end{array}$ & 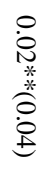 & $\begin{array}{l}\dot{0} \\
\dot{8} \\
\stackrel{0}{0} \\
\stackrel{N}{N}\end{array}$ & 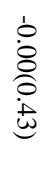 & & $\begin{array}{l}\dot{b} \\
\dot{+} \\
\stackrel{+}{0} \\
\dot{\vec{J}} \\
\mathbb{E}\end{array}$ & 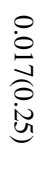 & $\begin{array}{l}\dot{0} \\
\dot{0} \\
\stackrel{0}{0} \\
\dot{a}\end{array}$ & $\begin{array}{l}\stackrel{0}{\dot{w}_{w}} \\
* \\
* \\
\stackrel{*}{0} \\
\stackrel{8}{8}\end{array}$ & & 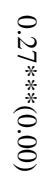 & 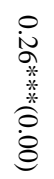 & 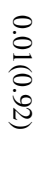 & $\begin{array}{l}\dot{b} \\
\dot{8} \\
\stackrel{0}{0} \\
\text { N } \\
\text { E }\end{array}$ & $\begin{array}{l}\stackrel{0}{0} \\
\dot{0} \\
\text { Oे } \\
\dot{+}\end{array}$ & $\begin{array}{l}\dot{0} \\
\dot{0} \\
\stackrel{0}{0} \\
\text { i্d }\end{array}$ & 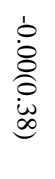 & $\begin{array}{l}\dot{0} \\
\dot{W} \\
\stackrel{0}{0} \\
\dot{\omega}\end{array}$ & $\begin{array}{l}\stackrel{0}{0} \\
\dot{0} \\
\dot{0} \\
\dot{\omega}\end{array}$ & $\vec{r}$ \\
\hline 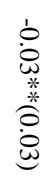 & $\begin{array}{l}\stackrel{0}{0} \\
\stackrel{0}{0} \\
\dot{\infty} \\
\dot{\infty}\end{array}$ & $\begin{array}{l}\stackrel{0}{0} \\
\text { Oे } \\
\text { î }\end{array}$ & & $\begin{array}{l}\stackrel{0}{\dot{0}} \\
\dot{0} \\
\text { 崩 }\end{array}$ & $\begin{array}{l}\dot{b} \\
\dot{0} \\
0 \\
\dot{0}\end{array}$ & 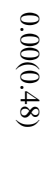 & $\begin{array}{l}\stackrel{0}{0} \\
\text { i } \\
\text { * } \\
\text { Oे } \\
\dot{0}\end{array}$ & & 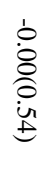 & 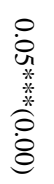 & 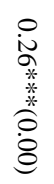 & 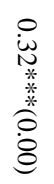 & & 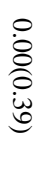 & $\begin{array}{l}\stackrel{0}{0} \\
\dot{0} \\
\stackrel{0}{0} \\
\dot{\tilde{N}}\end{array}$ & $\begin{array}{l}\stackrel{0}{0} \\
\stackrel{0}{0} \\
\stackrel{्}{J}\end{array}$ & 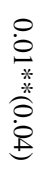 & $\begin{array}{l}\dot{0} \\
\dot{\delta} \\
0 \\
\dot{0} \\
\dot{0}\end{array}$ & $\begin{array}{l}\stackrel{0}{0} \\
\dot{W} \\
\infty \\
\stackrel{\infty}{0} \\
\text { N } \\
\text { N }\end{array}$ & 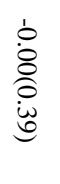 & $\overrightarrow{{ }^{\prime}}$ \\
\hline & & & & & & & $\tilde{\xi}^{\infty}$ & هి & $\begin{array}{l}\text { की } \\
\text { है } \\
\text { है }\end{array}$ & $\Phi$ & $\begin{array}{l}\infty \\
\tilde{b}_{0}^{\infty}\end{array}$ & $\tilde{\Sigma}$ & $\frac{\widetilde{8}}{8}$ & స్ & $\widetilde{E}$ & $\begin{array}{l}\tilde{c} \\
\tilde{b}_{0}\end{array}$ & $\vec{\omega}$ & $\vec{N}$ & $\precsim$ & $\varepsilon$ & 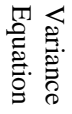 \\
\hline & & & & & & & 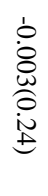 & $\begin{array}{l}\dot{0} \\
\dot{+} \\
* \\
* \\
\stackrel{*}{*} \\
\dot{0}\end{array}$ & \begin{tabular}{l}
$\stackrel{0}{0}$ \\
$\stackrel{0}{\omega}$ \\
\multirow{*}{*}{} \\
\multirow{*}{*}{} \\
$\stackrel{0}{0}$ \\
$\stackrel{8}{0}$
\end{tabular} & $\begin{array}{l}\dot{b} \\
\dot{0} \\
\stackrel{+}{0} \\
0 \\
\dot{E}\end{array}$ & & $\begin{array}{l}\stackrel{\ominus}{\varrho} \\
\text { * } \\
\stackrel{*}{*} \\
\dot{\theta}\end{array}$ & 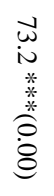 & $\begin{array}{l}\stackrel{N}{\circ} \\
\stackrel{0}{0} \\
\stackrel{\Xi}{\Xi}\end{array}$ & 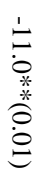 & & 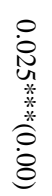 & $\begin{array}{l}\stackrel{0}{0} \\
\dot{\omega} \\
* \\
* \\
* \\
\stackrel{*}{0} \\
\dot{8}\end{array}$ & 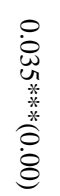 & 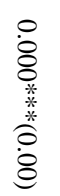 & 希 \\
\hline & & & & & & & 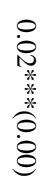 & $\begin{array}{l}\stackrel{0}{0} \\
\stackrel{0}{0} \\
\dot{0} \\
\stackrel{0}{0}\end{array}$ & & $\begin{array}{l}\stackrel{0}{0} \\
\stackrel{0}{0} \\
\stackrel{0}{0}\end{array}$ & $\begin{array}{l}\stackrel{0}{\circ} \\
\stackrel{8}{0} \\
\stackrel{0}{E}\end{array}$ & 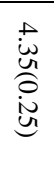 & 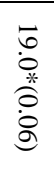 & & \begin{tabular}{l}
$\infty$ \\
$\stackrel{+}{ \pm}$ \\
\multirow{*}{*}{} \\
$\stackrel{0}{0}$ \\
$\dot{0}$
\end{tabular} & 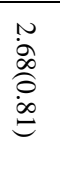 & 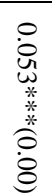 & 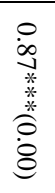 & \begin{tabular}{l}
$\stackrel{8}{0}$ \\
$\dot{+}$ \\
$*$ \\
$*$ \\
\multirow{*}{*}{} \\
$\dot{8}$ \\
$\dot{8}$
\end{tabular} & $\begin{array}{l}\stackrel{्}{8} \\
\dot{8} \\
\stackrel{*}{*} \\
\stackrel{*}{0} \\
\dot{d}\end{array}$ & 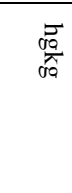 \\
\hline & & & & & & & 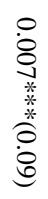 & & 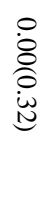 & $\begin{array}{l}\stackrel{0}{0} \\
\stackrel{0}{\omega} \\
* \\
* \\
\stackrel{*}{0} \\
\stackrel{0}{0}\end{array}$ & 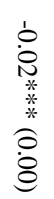 & 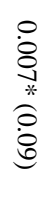 & & 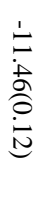 & 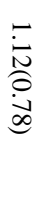 & $\begin{array}{l}u \\
\ddot{d} \\
\text { Oे } \\
\dot{g}\end{array}$ & 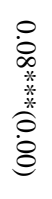 & 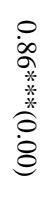 & 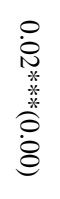 & 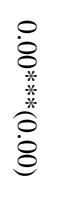 & $\breve{~}$ \\
\hline & & & & & & & \begin{tabular}{l}
0 \\
$\dot{0}$ \\
* \\
$*$ \\
\multirow{*}{*}{} \\
$\stackrel{0}{8}$ \\
0
\end{tabular} & 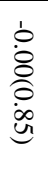 & $\begin{array}{l}\stackrel{0}{0} \\
\dot{0} \\
\text { Oे } \\
\text { N }\end{array}$ & & $\begin{array}{l}\stackrel{0}{0} \\
\dot{0} \\
\text { Oे } \\
\dot{0}\end{array}$ & $\begin{array}{l}\dot{0} \\
\dot{\delta} \\
\text { Oे } \\
\dot{\ddot{d}}\end{array}$ & 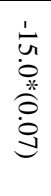 & 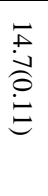 & 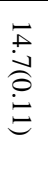 & \begin{tabular}{l}
$n$ \\
$\infty$ \\
in \\
$*$ \\
\multirow{2}{*}{} \\
0 \\
0 \\
0
\end{tabular} & 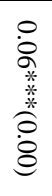 & 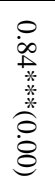 & 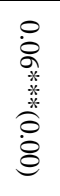 & 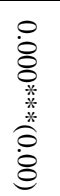 & $\vec{\Xi}$. \\
\hline
\end{tabular}

\section{EMPIRICAL RESULTS}

Table I and Table II highlights the value of correlation for the returns used in this study. All the markets are correlated which signifies the importance of this study. If markets are correlated then there is a chance that holidays in one market affect other markets. The multicollinearity problem in both types of returns (ordinary returns and intraday returns) has been tested separately through the correlation matrix using
Pearson's correlation test. The correlation result in both types indicates that there is no stern multicollinearity problem.

Table III summarizes the descriptive statistics and distributional properties of index of ordinary returns $\left(R_{t}^{c c}\right.$ close to close returns) and the intraday return ( $R_{t}^{o c}$ open to close return). The returns are slightly skewed. Furthermore surfeit kurtosis confirms the leptokurtic nature of the distributions of index return. 
TABLE IV (B): ESTIMATION- INTRADAY RETURN

\begin{tabular}{|c|c|c|c|c|c|c|c|c|c|c|c|c|c|c|c|c|c|c|c|c|c|}
\hline$\tilde{\Sigma}^{N}$ & $\stackrel{\overbrace{}}{\approx}$ & $\frac{\widetilde{c}}{\frac{6}{6}}$ & $\widetilde{\Xi}^{n}$ & $\begin{array}{l}\tilde{n}^{n} \\
\tilde{b}^{2}\end{array}$ & $D_{5}^{D}$ & ) & 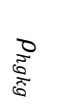 & D. & $\begin{array}{l}\text { D. } \\
\vdots \\
\vdots\end{array}$ & $\infty$ & ఫొ & 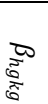 & $\underset{\Xi}{\Phi}$ & 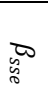 & $\stackrel{8}{ }$ & $\omega^{R}$ & $\beta$ & $\curvearrowright$ & $\theta$ & 8 & 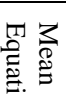 \\
\hline 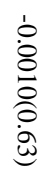 & $\begin{array}{l}\stackrel{0}{0} \\
\dot{8} \\
\grave{0} \\
\infty \\
\infty \\
\infty\end{array}$ & $\begin{array}{l}\dot{0} \\
\dot{8} \\
0 \\
0 \\
\stackrel{0}{0} \\
\text { N }\end{array}$ & 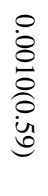 & & 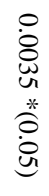 & 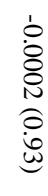 & 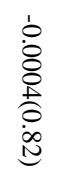 & $\begin{array}{l}\stackrel{0}{8} \\
\dot{0} \\
\stackrel{0}{0} \\
\dot{\hat{\sigma}}\end{array}$ & & \begin{tabular}{l}
$\dot{1}$ \\
$\dot{0}$ \\
\multirow{2}{*}{} \\
$*$ \\
\multirow{*}{*}{} \\
$\stackrel{8}{8}$
\end{tabular} & 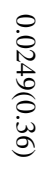 & 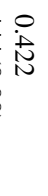 & 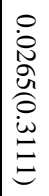 & & 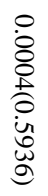 & \begin{tabular}{l}
$\dot{0}$ \\
$\dot{8}$ \\
$\stackrel{0}{0}$ \\
\multirow{*}{*}{} \\
$\stackrel{*}{*}$ \\
$\dot{+}$
\end{tabular} & 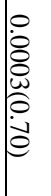 & $\begin{array}{l}\dot{0} \\
\dot{8} \\
\stackrel{0}{0} \\
\dot{0}\end{array}$ & 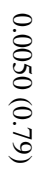 & 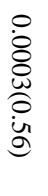 & $\overrightarrow{F_{\tilde{C}}}$ \\
\hline 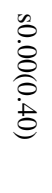 & 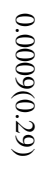 & & $\begin{array}{l}\dot{0} \\
\dot{8} \\
\stackrel{0}{0} \\
\text { i্ }\end{array}$ & $\begin{array}{l}\dot{0} \\
\dot{8} \\
8 \\
0 \\
\vdots\end{array}$ & $\begin{array}{l}\stackrel{\circ}{8} \\
\stackrel{0}{0} \\
\stackrel{0}{\Xi}\end{array}$ & $\begin{array}{l}\dot{b} \\
\dot{8} \\
\stackrel{0}{0} \\
\dot{d}\end{array}$ & & $\begin{array}{l}\stackrel{\circ}{8} \\
\stackrel{8}{0} \\
\stackrel{0}{0} \\
\stackrel{N}{E}\end{array}$ & 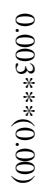 & 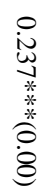 & 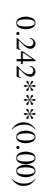 & & 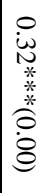 & 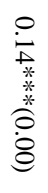 & $\begin{array}{l}\dot{b} \\
\dot{8} \\
\stackrel{0}{0} \\
\text { : } \\
0\end{array}$ & 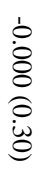 & 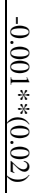 & 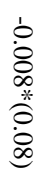 & 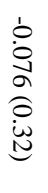 & $\begin{array}{l}\stackrel{0}{8} \\
\dot{8} \\
\stackrel{*}{*} \\
\stackrel{0}{0} \\
\dot{0}\end{array}$ & $\overrightarrow{\vec{T}^{0}}$ \\
\hline $\begin{array}{l}\stackrel{0}{0} \\
\dot{0} \\
\dot{0} \\
\dot{U}\end{array}$ & & 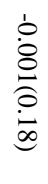 & 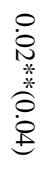 & $\begin{array}{l}\dot{0} \\
\dot{8} \\
\stackrel{0}{0} \\
\stackrel{N}{N}\end{array}$ & $\begin{array}{l}\dot{0} \\
\dot{8} \\
\stackrel{0}{0} \\
\stackrel{+}{\omega}\end{array}$ & & $\begin{array}{l}\dot{0} \\
\dot{+} \\
\stackrel{+}{0} \\
\dot{\vec{A}} \\
\stackrel{ \pm}{E}\end{array}$ & 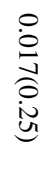 & 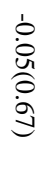 & 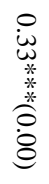 & & 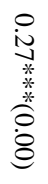 & 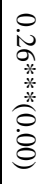 & 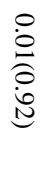 & $\begin{array}{l}\dot{1} \\
\dot{0} \\
\stackrel{0}{0} \\
\text { in } \\
\pm\end{array}$ & $\begin{array}{l}\stackrel{0}{\circ} \\
\dot{0} \\
0 \\
\dot{0} \\
\dot{\infty}\end{array}$ & 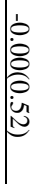 & $\begin{array}{l}\dot{b} \\
\dot{ᄋ} \\
\stackrel{0}{0} \\
\dot{\omega} \\
\infty\end{array}$ & $\begin{array}{l}\dot{b} \\
\dot{\omega} \\
\stackrel{\omega}{0} \\
\stackrel{0}{0}\end{array}$ & $\begin{array}{l}\stackrel{0}{\circ} \\
\stackrel{8}{0} \\
\stackrel{0}{\infty} \\
\underbrace{\infty}\end{array}$ & 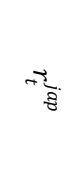 \\
\hline 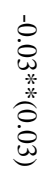 & $\begin{array}{l}\stackrel{0}{8} \\
\stackrel{0}{0} \\
\stackrel{\infty}{\infty}\end{array}$ & $\begin{array}{l}\stackrel{0}{8} \\
\stackrel{8}{0} \\
\text { 잉 }\end{array}$ & & 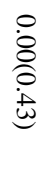 & 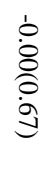 & $\begin{array}{l}\stackrel{\circ}{8} \\
\stackrel{0}{0} \\
\stackrel{+}{\infty}\end{array}$ & \begin{tabular}{l}
0 \\
$\dot{0}$ \\
\multirow{3}{*}{} \\
$\stackrel{0}{0}$ \\
$\dot{0}$
\end{tabular} & & 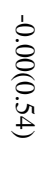 & 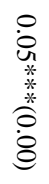 & 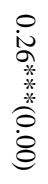 & 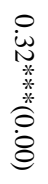 & & 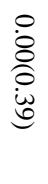 & 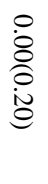 & $\begin{array}{l}\stackrel{\circ}{\circ} \\
\stackrel{O}{0} \\
\stackrel{\text { Ju }}{ }\end{array}$ & 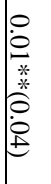 & $\begin{array}{l}\dot{1} \\
\dot{0} \\
0 \\
0 \\
0 \\
0\end{array}$ & 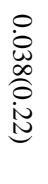 & $\begin{array}{l}\dot{b} \\
\dot{0} \\
\stackrel{0}{0} \\
\dot{\omega}=0\end{array}$ & $\overrightarrow{\vec{g}}$ \\
\hline & & & & & & & $\tilde{\xi}^{0}$ & $\stackrel{10}{\text { छे }}$ & 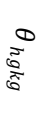 & $\stackrel{D}{\Phi}$ & $\begin{array}{l}\infty \\
6 \\
6 \\
6\end{array}$ & $\tilde{\xi}$ & ๕ृ & $\begin{array}{l}\text { वृ } \\
\text { है }\end{array}$ & ฐ & $\begin{array}{l}\tilde{c}^{2} \\
\omega^{2}\end{array}$ & $\vec{\omega}$ & $\stackrel{\sim}{\sim}$ & 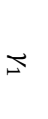 & $\varepsilon$ & 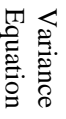 \\
\hline & & & & & & & 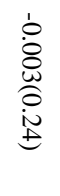 & 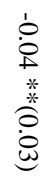 & $\begin{array}{l}\stackrel{0}{0} \\
\dot{0} \\
w \\
* \\
* \\
* \\
0 \\
\stackrel{8}{8}\end{array}$ & $\begin{array}{l}\dot{0} \\
\dot{8} \\
+ \\
\\
\dot{1}\end{array}$ & & $\begin{array}{l}\stackrel{0}{0} \\
\stackrel{*}{*} \\
\stackrel{*}{*} \\
\stackrel{0}{\Theta}\end{array}$ & 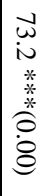 & $\begin{array}{l}\text { No } \\
\stackrel{\bigcirc}{\circ} \\
\stackrel{⿹}{\Xi}\end{array}$ & 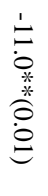 & & 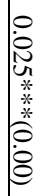 & 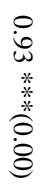 & 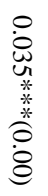 & 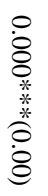 & $\tilde{z}$ \\
\hline & & & & & & & 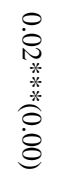 & $\begin{array}{l}\stackrel{0}{8} \\
\stackrel{0}{0} \\
\text { o } \\
\stackrel{0}{0}\end{array}$ & & $\begin{array}{l}\stackrel{0}{\circ} \\
\stackrel{0}{0} \\
\stackrel{0}{0}\end{array}$ & $\begin{array}{l}\stackrel{0}{8} \\
\stackrel{0}{0} \\
\stackrel{0}{ \pm} \\
\text { છ }\end{array}$ & 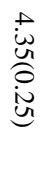 & $\begin{array}{l}\vec{b} \\
\stackrel{*}{\circ} \\
\stackrel{*}{\circ} \\
\dot{0}\end{array}$ & & 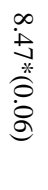 & $\begin{array}{l}\stackrel{N}{\mathscr{\infty}} \\
\stackrel{\infty}{O} \\
\stackrel{\infty}{=}\end{array}$ & 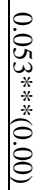 & 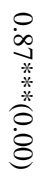 & 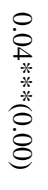 & 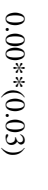 & 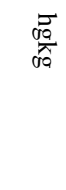 \\
\hline & & & & & & & 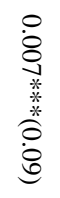 & & 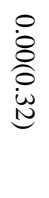 & $\begin{array}{l}\stackrel{0}{0} \\
\stackrel{0}{0} \\
* \\
* \\
* \\
\stackrel{*}{*} \\
\stackrel{8}{8}\end{array}$ & 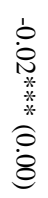 & 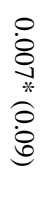 & & 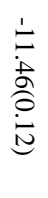 & 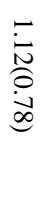 & 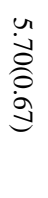 & 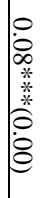 & 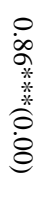 & 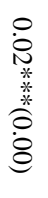 & 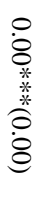 & 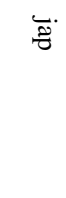 \\
\hline & & & & & & & 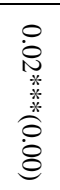 & $\begin{array}{l}\dot{b} \\
\dot{8} \\
\stackrel{0}{0} \\
\dot{0} \\
\dot{\infty}\end{array}$ & $\begin{array}{l}\stackrel{0}{8} \\
\stackrel{0}{0} \\
\text { Oे } \\
\text { N }\end{array}$ & & 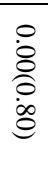 & $\begin{array}{l}\dot{b} \\
\dot{0} \\
\stackrel{0}{0} \\
\stackrel{d}{0}\end{array}$ & 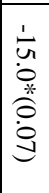 & 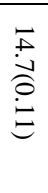 & $\begin{array}{l}\stackrel{\vec{D}}{\vec{J}} \\
\stackrel{0}{\Xi}\end{array}$ & 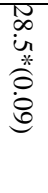 & 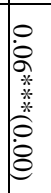 & 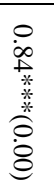 & 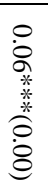 & \begin{tabular}{l}
$\stackrel{0}{8}$ \\
$\dot{8}$ \\
\multirow{*}{*}{} \\
$\stackrel{*}{*}$ \\
$\dot{8}$ \\
$\dot{8}$
\end{tabular} & $\vec{\Xi}$. \\
\hline
\end{tabular}

Thus all the returns can be used to test the model. Parameter estimates of East Asian stock market for both types of returns showing mean and variance equation is summarized in Table IV (a) \& IV (b). Analyzing coefficients estimates of mean equation, Chinese market shows negative Day-of-the-week effect for Thursday [11]. In Hong Kong market we found significant negative Day-of-the-week effect for Wednesday in both types of returns but negative effect of Tuesday is statistically significant in ordinary returns only, inconsistent with previous literature [12]. In Taiwan market we establish significant positive Day-of-the-week effect for Wednesday [13]. In Chinese market, for intraday returns Hong Kong and Taiwan is showing positive significant effect with no effect of international spillover but in ordinary returns only Hong Kong is showing positive significant effect and negative effect of global spillover in Chinese market. In case of Hong Kong, area and international spillover coefficients are significant but extent of the spillover effect from area and international markets varies. For Japanese market, intraday returns these coefficients have significant impact with the variability of magnitude These results are consistent in ordinary returns with the exception of Chinese market. Coefficients estimates of (Taiwan) mean equation shows that in intraday returns area and international spillover coefficients are found to be significant. But the intensity of regional and international spill over is different. These results 
are consistent in ordinary returns with the exception of Chinese market. The sign of news may have an impact on price movements [9].

Chinese market shows statistical significant effect of lag of positive closure in US market for ordinary return whereas lag of positive closure of Japanese market is having negative significant effect for intraday return. In Hong Kong market statistical significant effect of lag of positive closure in Chinese market for both types of returns. But intraday returns have an additional impact of second lagged positive closure of USA and negative closure of Chinese market. Japanese market shows statistical positive significant effect of lagged negative closure in Taiwan market for ordinary return whereas lagged negative closure of Taiwan and Hong Kong market is having positive and negative significant effect for intraday return respectively. Taiwanese market shows statistically significant effect of second lagged negative closure in USA market for ordinary return and intraday return, as there is time zone difference of 12 hours in East Asian market and USA. Lag of positive closure Hong Kong market is having positive significant effect in both cases. These results are consistent with previous studies [7].Turning towards the variance equation. Positive coefficient of $\gamma_{1}$ in both types of returns for four selected East Asian market proposes that abrupt stock returns $\left(\varepsilon_{t-1}^{2}\right)$ upsurge the riskiness in stock market.

Additionally, positive and significant values of $\left(\gamma_{2}\right)$ expresses that uncertainty in the East Asian stock market is highly tenacious. As $\left(\gamma_{3}\right)$ is statistically significant and positive, we can clinch that non positive information in these markets has more effect in contrast to positive information of the same magnitude. In Chinese market, volatility spillover effect of Hong Kong and Japan remain significant in both types of returns. First lag of Taiwan holiday has negative significant effect on volatility in both types of return. First lag of Japanese holidays has positive effect on the volatility of intraday and ordinary return. But the second lag of USA is statistically significant in ordinary returns only.

In Hong Kong market international volatility spillover effect is significant in both types of return. In regional volatility spillover effect only Taiwan is showing significant effect. Second lag effect of US holiday is negatively significant in intraday return. Lag effect of Japanese holiday is statistical significant in both cases. But lag effect of Chinese holiday has positive significant effect for intraday returns. Japanese market in both types of returns, regional volatility spillover effect remains significant in intraday return. There is positive spillover effect of Taiwan and negative spillover effect of China along with positive global spillover effect. Second lag effect of USA holiday is statistically significant in ordinary return. But in intraday return lag effect of holiday in China and Taiwan are significant. For Taiwanese market there is positive global spillover effect in ordinary as well in intraday return. In regional volatility spillover effect Hong- Kong is statistically significant in intraday return. Second lag effect of USA holiday is statistically significant in intraday return. Lag effect of Japanese holiday is significant in both types of returns. But lag effect of holiday in China is significant in ordinary return.

\section{CONCLUSION}

This paper contributes to the debate on effect of holidays of interconnected stock markets on one another. Empirical results showed that stock markets in East Asian region are having statistically significant correlation. Also there are return and volatility spillovers from one market to other. But mainland Chinese stock market being biggest market in the region has minimum spillovers from other markets. Our empirical results on lagged effect of holidays show that information inflow and flow of investment due to portfolio adjustment plays a vital role in market activities. If one market is closed in the region it has effect on the following days return due to portfolio adjustment. Not only returns are being affected but volatility of the market as well. Even there is different effect of holiday which is after a positive or negative return day. Our results also shows that in the East Asian economies investors are not good news seekers instead investors react strongly to negative information in comparison to positive news of same magnitude. Results provided by this paper have implications for international investors. It is of immense importance for an investor to consider the holidays of the interlinked stock markets when investing in a particular market of a region as holidays not only affect the returns of the portfolios but risk as well. Future research can be conducted on volume data as well as spread of ask and bid prices so that effect of holidays can be studied on other dynamics of market.

\section{REFERENCES}

[1] G. Bekaert and C. Harvey, "Emerging equity market volatility," Journal of Financial Economics, vol. 43, pp. 29-77, 1997.

[2] East Asia and Pacific. (2013). [Online]. Available: http://www.worldbank.org/en/region/eap

[3] E. Fama, "Efficient capital markets: A review of theory and empirical work," The Journal of Finance, vol. 25, pp. 383-417, 1970.

[4] J. Lakonishok and S. Smidt, "Are seasonal anomalies real? A ninety-year perspective," Review of Financial Studies, vol. 1, pp. 403-425, 1988.

[5] X. Cao, I. M. Premachandra, G. S. Bharba, and Y. P. Tang, "Firm size and the pre-holiday effect in New Zealand," International Research Journal of Finance and Economics, vol. 32, pp. 171-187. 2009.

[6] G. K. Marrett and A. C. Worthington, "An empirical note on the holiday effect in the Australian stock market," Applied Financial Letters, vol.16, pp. 1769-1772, 2009.

[7] E. Baumohl and T. Vyrost, "Stock market integration: Granger causality testing with respect to nonsynchronous trading effects," Czech Journal of Economics and Finance, vol. 60, pp. 414-425, 2010

[8] S. Lin and M. Tamvakis, "Spillover effects in energy futures markets," Energy Economics, vol. 23, pp.43-56, 2001.

[9] J. Casado, L. Muga, and R. S. Maria, "The effect of US holidays on the European markets: When the cat's away," Accounting and Finance, vol. 53, pp. 111-136, 2013.

[10] L. Glosten, R. Jagnnathan, and D. Runkle, "On the relation between the expected value and volatility of the nominal excess return on stocks," Journal of Finance, vol. 48, pp. 1770-1801, 1993.

[11] T. M. Bohl, S. Michael, and P. L. Siklos, "Stock returns seasonality's and investor structure: Evidence from China's B-share markets," Center for Quantitative Economics, vol. 27, pp. 433-454, 2009.

[12] R. J. Doyle and H. C. Chen, "The wandering weekday effect in major stock markets," Journal of Banking and Finance, vol. 33, pp. 1388-1399, 2009.

[13] M. C. Kea, Y. C. Chiang, and T. L. Liao, "Day-of-the-week effect in the Taiwan foreign exchange market," Journal of Banking and Finance, vol. 31, pp. 2847-2865, 2007. 
Beenish Bashir was born in Islamabad, Pakistan, on $11^{\text {th }}$ November, 1988. She received her master in Accounts and Finance from University of Lahore, Pakistan in 2012. She is currently doing her MPhil in Finance from National University of Sciences and Technology (NUST), Islamabad, Pakistan.The author's major areas of research are financial markets and Islamic finance. Ms. Beenish Bashir is Gold Medalist in her master degree.

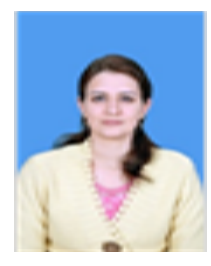

Shumaila Zeb was born in Islamabad Pakistan, on $6^{\text {th }}$ June, 1985. She received her master in Business Administration (MBA-Finance) from National University of Modern Languages, Pakistan in 2009. She is currently doing her MPhil (Finance) from National University of Sciences and Technology (NUST), Islamabad, Pakistan. The author's major areas of research are Islamic Finance and financial markets. She is serving as a visiting Assistant Professor in Arid Agriculture University, Pakistan. Also worked as Technical writer Iwebpros for one year March 2010 from Dec 2005 to Dec 2007. 